\title{
Influencia de la alta velocidad ferroviaria en la elección del destino turístico según el origen de los viajeros. El caso de la Costa Dorada en Cataluña*
}

\author{
Òscar Saladié \\ Aaron Gutiérrez \\ Salvador Anton Clavé \\ Universitat Rovira i Virgili. Departament de Geografia \\ oscar.saladie@urv.cat \\ aaron.gutierrez@urv.cat \\ salvador.anton@urv.cat
}

\section{Resum}

La llegada de la alta velocidad ferroviaria (AVF) a Tarragona en 2006 añade otra opción de transporte para los turistas que se desplazan hasta un destino litoral como es la Costa Dorada (Cataluña). Mediante la aplicación de un método probabilístico causal hemos calculado el incremento en la probabilidad de que este destino turístico haya sido visitado debido a la existencia de la estación de AVF Camp de Tarragona. El trabajo se ha basado en los resultados de una encuesta a pasajeros españoles de la AVF que han realizado sus vacaciones en la Costa Dorada. Los resultados muestran que el incremento de probabilidad es mayor entre aquellos que residen en regiones más alejadas. Este incremento es de un $12 \%$ entre los pasajeros españoles de la AVF procedentes de Aragón y un 16,6\% entre los de Madrid, regiones que representan casi dos terceras partes de los pasajeros de la muestra. Se ha demostrado también la existencia de importantes diferencias de carácter sociodemográfico y en relación con las características del viaje y de la estancia en función de la región de origen de los pasajeros.

Palabras clave: alta velocidad ferroviaria; elección del destino turístico; origen de los turistas; Costa Dorada; Cataluña

* Este estudio ha contado con el apoyo del Ministerio de Economía y Competitividad del Gobierno de España (proyecto MOVETUR CSO2014-51784-R), de la Cátedra Dow/URV de Desarrollo Sostenible y del Programa Serra Húnter de la Generalitat de Catalunya. Como resultado del proyecto de investigación mencionado han aparecido recientemente otros dos artículos que utilizan los mismos datos primarios. En 2016 apareció una contribución en la revista Belgeo (Saladié et al., 2016) que analiza si los pasajeros que llegaban en tren a la Costa Dorada habían visitado previamente o no este destino. En marzo de 2018 apareció en Boletín de la $A G E$ un artículo cuyo objeto es la influencia que la estación de alta velocidad Camp de Tarragona ejerce en la elección de la Costa Dorada como destino de vacaciones (Gutiérrez et al., 2018). El presente artículo introduce la influencia diferencial que el origen geográfico regional tiene en la elección del viaje en tren a la Costa Dorada de los turistas españoles. 
Resum. Influència de l'alta velocitat ferroviària en l'elecció de la destinació turística segons l'origen dels viatgers. El cas de la Costa Daurada a Catalunya

L'arribada de l'alta velocitat ferroviària (AVF) a Tarragona l'any 2006 afegeix una altra opció de transport per als turistes que es desplacen fins a una destinació litoral com és la Costa Daurada (Catalunya). Mitjançant l'aplicació d'un mètode probabilístic causal hem calculat l'increment en la probabilitat que aquesta destinació turística hagi estat visitada degut a l'existència de l'estació d'AVF. L'estudi s'ha basat en els resultats d'una enquesta a passatgers espanyols que han fet les seves vacances a la Costa Daurada. Els resultats mostren que l'increment de probabilitat és més gran entre els residents en regions més allunyades. Aquest increment és d'un 12\% entre els passatgers espanyols procedents d'Aragó i un d'un 16,6\% entre els de Madrid, regions que representen gairebé dues terceres parts dels passatgers de la mostra. També s'ha demostrat que hi ha importants diferències de caràcter sociodemogràfic i en relació amb les característiques del viatge i de l'estada.

Paraules clau: alta velocitat ferroviària; elecció de la destinació turística; origen dels turistes; Costa Daurada; Catalunya

Résumé. Influence du train à grande vitesse sur le choix de la destination touristique en fonction de la région d'origine des voyageurs. Le cas de la Costa Daurada en Catalogne

L'arrivée du train à grande vitesse (TGV) à Tarragona en 2006 ajoute une autre option de transport pour les touristes qui veulent se déplacer vers une destination touristique comme celle de la Costa Daurada (en Catalogne). Nous avons appliqué une méthode probabiliste de causalité pour calculer l'augmentation de la probabilité de fréquentation de cette destination touristique grâce à l'existence de la gare du TGV, en fonction de la région d'origine des voyageurs espagnols en vacances sur la Costa Daurada. Les résultats nous montrent que la probabilité est plus élevée pour les touristes qui habitent dans les régions les plus éloignées: $12 \%$ pour ceux d'Aragon et 16,6\% pour ceux de Madrid. Ces deux régions sont les lieux d'origine de presque les deux tiers des voyageurs de l'échantillon. Nous avons aussi relevé des différences importantes dans les caractéristiques sociodémographiques des voyageurs ainsi que dans les caractéristiques du voyage et du séjour.

Mots-clés: train à grande vitesse; choix de la destination touristique; provenance des touristes; Costa Daurada; Catalogne

Abstract. Influence of high speed rail services on tourist's destination choice according to passengers' origin: The case of the Costa Daurada in Catalonia

The arrival of high-speed rail (HSR) services in Tarragona in 2006 adds another transportation option for tourists who travel to a coastal destination such as the Costa Daurada of Catalonia. The increased likelihood of visiting this destination due to the existence of a HSR station has been analyzed using a causal probabilistic method. The study is based on the results of a survey of Spanish HSR passengers who spend their holidays on the Costa Daurada. The results show that the increase in the probability is higher among tourists from remote regions. Specifically, it is $16.6 \%$ higher for tourists from Madrid and 12\% higher for tourists from Aragon. These two regions account for almost two-thirds of the passengers in the sample. There are also differences in sociodemographic, trip and stay characteristics related to the tourist's origin.

Keywords: high-speed rail; tourist's destination choice; origin of tourists; Costa Daurada; Catalonia 


\author{
Sumario \\ 1. Introducción 4. Resultados \\ 2. Área de estudio 5. Conclusiones \\ 3. Datos y metodología Referencias bibliográficas
}

\title{
1. Introducción
}

Los factores que influyen en la elección del destino turístico son de naturaleza diversa y están vinculados tanto a los turistas (Um y Crompton, 1990), como a los destinos (Crouch y Ritchie, 2003). Entre los primeros hay que diferenciar entre los de carácter interno (p. ej. características sociodemográficas de los turistas y sus actitudes) y los de carácter externo (p. ej. estímulos sociales recibidos por los turistas y políticas de promoción de los destinos). Entre los segundos se encuentran los recursos y atractivos del destino o la mayor o menor accesibilidad al mismo. El transporte, entendido como el sistema que permite a un turista desplazarse desde su lugar de origen hasta el destino escogido, juega un papel clave en el posicionamiento de los destinos turísticos (Enright y Newton, 2004; Khadaroo y Seetanah, 2008; Cracolici y Nijkamp, 2008; Gossling et al., 2009; Page y Connell, 2009) de la misma manera que el desarrollo y la competitividad de los destinos están condicionados por las infraestructuras de transporte que posibilitan o facilitan una mayor accesibilidad y conectividad (Lumsdon y Page, 2004). De esta manera el transporte está considerado como uno de los factores determinantes en la elección del destino turístico (Decrop y Snelders, 2004).

La distancia existente entre dos territorios ha incidido tradicionalmente en sus interrelaciones (Eldridge y Jones, 1991), disminuyendo las segundas a medida que aumentaba la primera. El turismo no es una excepción tal como, hace ya cuatro décadas, Miossec (1977) modelizó al vincular la mayor o menor llegada de turistas a un destino a los costes y duración, tanto del viaje hasta el destino, como de la estancia en el mismo. De esta manera, para un destino determinado, la cantidad de turistas procedentes de un mismo origen disminuye a medida que aumenta la distancia donde se encuentra el destino. A su vez, diferentes distancias implican diferentes medios de transporte protagonistas. En términos generales, el vehículo particular es el modo preferente en desplazamientos cortos; mientras que el transporte aéreo es el protagonista en las distancias largas. El ferrocarril convencional puede competir, en términos de coste y eficiencia, con el vehículo particular en distancias medias (y cortasmedias). Y el tren de alta velocidad con el avión en distancias medias-largas (Albalate et al. 2015, Ureña et al., 2009).

La distancia entre el lugar de origen y el destino, vinculada al tiempo empleado en recorrerla, aún juega un papel muy importante en la elección del destino y en la mayor o menor llegada de turistas (Bull, 1991; Prideaux, 2002; Duval, 2007; Mazanec et al., 2007; Lee et al., 2012; Dickinson y Peeters, 
2014; Hooper, 2015). Esta influencia no únicamente está relacionada con la distancia física o el tiempo necesario en recorrerla, sino que también hay que tener en cuenta los costes atribuibles al desplazamiento, que acostumbran a ser mayores cuanto mayor es la distancia (Pirie, 2009; Lee et al., 2012). Ahora bien, la motivación del viaje de los turistas (p. ej. visitar a amigos o familiares) (McKercher y Lew, 2003) o los propios atributos del destino (Lee et al., 2012) pueden incorporar matices en la relación entre distancia y llegada de turistas. La distancia entre el destino turístico y el lugar de origen de los viajeros también influye en la duración de la estancia. La estancia en el destino es mayor entre los turistas procedentes de lugares más alejados (Wu y Carson, 2008; Thrane, 2012; Wang et al., 2012; Alén et al., 2014). Esta constatación es importante ya que, en términos generales, la duración de la estancia de los turistas suele ser una variable estratégica desde el punto de vista de la gestión de los destinos (Alegre y Pou, 2006; Gokovali et al., 2007; Gomes de Menezes et al., 2008; Salmasi et al., 2012; Ferrer-Rosell et al., 2014).

La alta velocidad ferroviaria (AVF) es un medio de transporte relativamente nuevo que está contribuyendo a mejorar la accesibilidad de los lugares en tanto que destinos turísticos ya que, en muchos casos, reduce el tiempo de viaje (Gutiérrez, 2004; Masson y Petiot, 2009; Ortega et al., 2012). Albalate y Bel (2011) consideran que existen cuatro grandes motivaciones que justifican la construcción de la red de AVF: (I) solucionar problemas de congestión; (II) conectar áreas industriales con centros de distribución y de transporte internacional; (III) promover la equidad y el desarrollo territorial y (IV) proporcionar una alternativa al transporte aéreo en países donde la geografía proporciona ventajas competitivas para el ferrocarril. En relación con este último punto, diferentes investigaciones han analizado la competencia entre el transporte aéreo y la AVF (Clewlow et al., 2014; Dobruszkes et al., 2014; Albalate et al., 2015). La conclusión general es que se trata de un medio de transporte más competitivo que el avión o la carretera cuando se trata de viajes de media distancia (entre 200 y $700 \mathrm{~km}$ ).

Diferentes estudios apuntan una progresiva diversificación del perfil de pasajeros y motivo de los viajes en AVF, especialmente en aquellos territorios que hace más tiempo que cuentan con estos servicios (Santos et al., 2007; Garmendia et al., 2011). Así, además de los habituales desplazamientos por motivos profesionales, la AVF también ha atraído viajes vinculados al ocio y el turismo. Ello abre nuevas oportunidades en este sector (Masson y Petiot, 2009; Coronado et al., 2012; Bazin et al., 2013), aunque ello ha derivado en la generación de expectativas, habitualmente sobredimensionadas, vinculadas a las posibilidades de dinamización turística, y económica en general, asociadas a la llegada de la AVF a un territorio (Bellet y Gutiérrez, 2011; Feliu, 2012).

Los estudios sobre las relaciones entre la alta velocidad ferroviaria y el turismo están recibiendo un importante impulso en los últimos años, especialmente en el caso del turismo urbano (Masson y Petiot, 2009; Bazin et al., 2010; Bellet et al., 2012; Feliu, 2012; Garmendia et al., 2012; Bazin et al., 2013; Delaplace et al., 2014; Pagliara et al., 2015) y, en menor medida, de los 
destinos turísticos litorales (Anton Clavé et al., 2015a; Ortuño et al., 2015; Saladié et al., 2016). Pocos son los estudios que han analizado los efectos de la introducción de los servicios de alta velocidad ferroviaria en la elección del destino turístico. Entre estos destacan los realizados por Delaplace et al. (2014) y Pagliara et al. (2015), que analizan los casos de grandes capitales europeas como son París-Roma y Madrid, respectivamente.

Tomando como punto de partida la información anterior, los objetivos del artículo son (i) analizar las diferencias de carácter sociodemográfico y de las características de la estancia de los turistas de las diferentes regiones españolas que han llegado a la Costa Dorada mediante la AVF; y (ii) determinar la desigual influencia que ejerce la existencia de la estación de AVF Camp de Tarragona en la elección de la Costa Dorada como destino turístico entre los españoles llegados con AVF de las diferentes regiones del Estado. Es decir, se pretende confrontar las diferencias, en términos de incremento de probabilidad de visitar el destino, entre las diferentes regiones de origen de los turistas.

Los resultados de este segundo objetivo no se han obtenido en base a una respuesta binaria (sí o no) a la pregunta sobre la influencia de la estación de AVF, como es en el caso de Delaplace (2014); ni tampoco en base a la elección de una razón entre un conjunto de posibles respuestas, como es en el caso de Pagliara et al. (2015). Hemos aplicado un método probabilístico causal desarrollado por Young et al. (2005), que permite determinar el efecto que la exposición a cierta información o productos (una estación de AVF) genera en la decisión de consumir dichos productos (escoger un determinado destino turístico). En estudios vinculados con el turismo, esta metodología fue aplicada por Fernandez Young y Young (2008) para analizar el efecto de las películas en las visitas de los turistas a determinados territorios, por Young et al. (2010) para el turismo cultural asociado a los mercados en las ciudades, y por Saladié et al. (2014) y Anton Clavé et al. (2015b) para cuantificar la influencia ejercida por una línea aérea de bajo coste en la elección del destino turístico. Fuera del ámbito turístico, también es la metodología escogida por Saladié y Pla (2015) y Saladié y Santos-Lacueva (2016) para determinar el incremento en la probabilidad de mejora de la recogida selectiva de los residuos domésticos atribuible a las campañas de sensibilización.

El estudio está organizado de la siguiente manera. En primer lugar (apartado 2) se describe el contexto territorial, tanto por lo que respecta al destino turístico (Costa Dorada), como a la estación de AVF Camp de Tarragona. A continuación (apartado 3) se detallan los datos utilizados y la metodología aplicada. El análisis y la discusión de los resultados, en relación con los dos objetivos planteados, se encuentran en el apartado 4. El estudio finaliza con las conclusiones y las referencias bibliográficas.

\section{2. Área de estudio}

La Costa Dorada, en la provincia de Tarragona (figura 1), es uno de los principales destinos turísticos de Cataluña. Recibió más de 4,5 millones de turistas 
Figura 1. Área de estudio: la Costa Dorada en la provincia de Tarragona

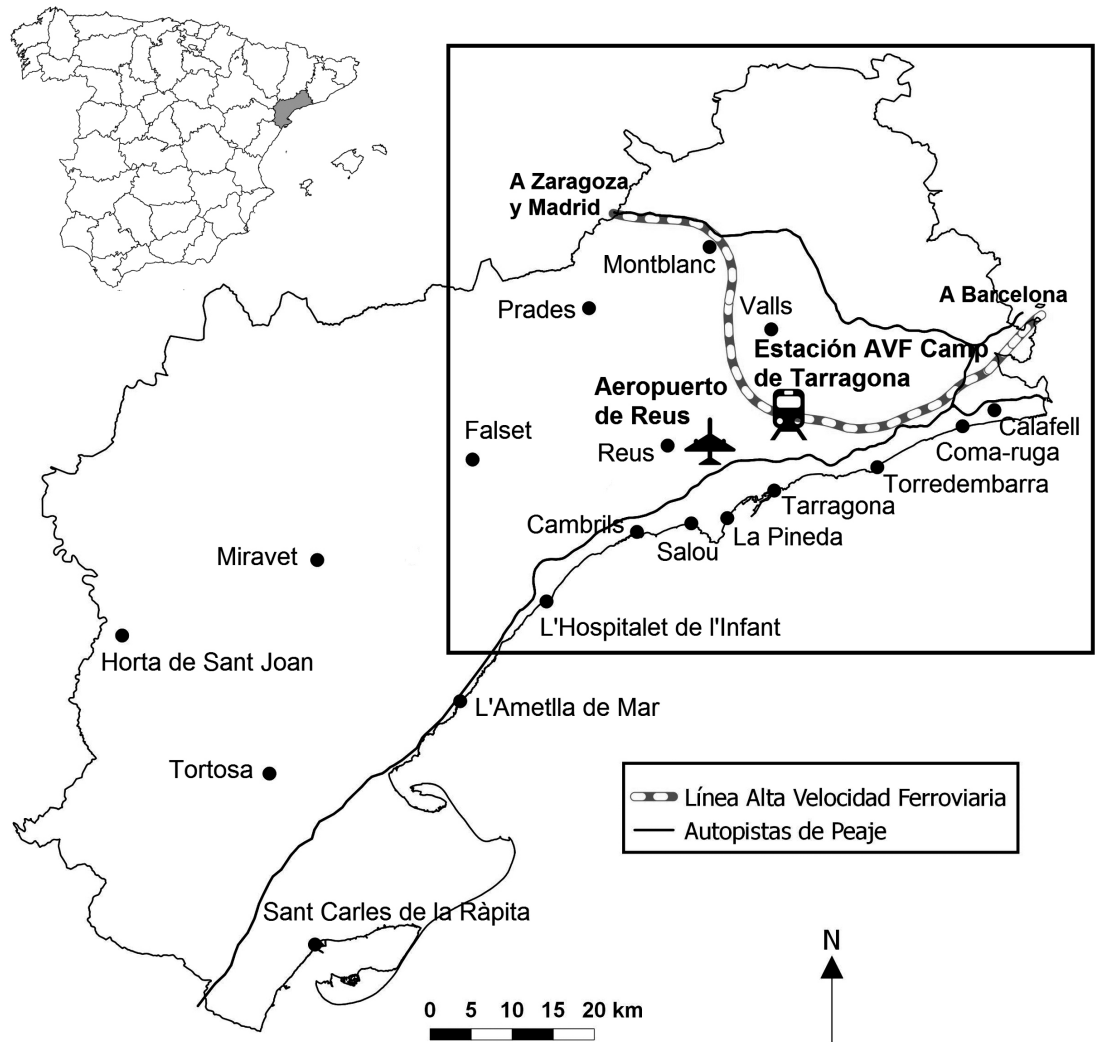

Fuente: elaboración propia.

durante el año 2014. De acuerdo con los datos del Observatorio de la Fundación de Estudios Turísticos de la Costa Dorada, estos turistas realizaron el desplazamiento desde su lugar de origen mayoritariamente mediante vehículo privado $(59 \%)$, seguidos por los que tomaron un avión (26\%). Ya a mucha distancia se encuentra el autobús (7\%), el tren (5\%) y otros medios de transporte $(3 \%)$. No obstante, la distribución varía sustancialmente si sólo se tienen en cuenta los turistas españoles, que representan un 57\% del total. La gran mayoría (75\%) se desplaza en vehículo privado. El tren se encuentra en segunda posición, con un $9 \%$ ( $5 \%$ trenes convencionales y $4 \%$ AVF), seguidos del autobús (8\%), el avión (7\%) y otros modos de transporte (1\%) (Gutiérrez y Miravet, 2016).

El territorio está bien conectado mediante autopistas de peaje: la autopista AP7 permite la conexión con Valencia y el resto del arco mediterráneo español en dirección suroeste, y con Barcelona y la frontera francesa en dirección nordeste; mientras que la autopista AP2 permite la conexión con 
Madrid, Zaragoza y el corredor del Ebro en dirección oeste. Por lo que respecta al transporte aéreo, la infraestructura más cercana es el aeropuerto de Reus, localizado a escasos $10 \mathrm{~km}$ de la ciudad de Tarragona y de la denominada Costa Dorada Central, que comprende Cambrils, Salou y La Pineda (núcleo marítimo de Vila-seca), de carácter regional y con un funcionamiento marcado por la estacionalidad. El aeropuerto de Barcelona-El Prat es también un punto importante de llegada de turistas que tienen como destino la Costa Dorada. Se encuentra a unos $100 \mathrm{~km}$ de Salou (el principal núcleo turístico del área).

Tarragona, Reus y otras muchas localidades del área de estudio disponen de estación de ferrocarril convencional. A su vez, en diciembre de 2006 entró en funcionamiento la estación de AVF Camp de Tarragona. Es una estación periférica, situada a unos $15 \mathrm{~km}$ al norte de la ciudad de Tarragona (figura 1) y a unos $20 \mathrm{~km}$ de Salou. Es un nodo intermedio de la línea de alta velocidad entre Madrid, Barcelona y la frontera francesa. En el año 2014 contó con más de 750 mil viajeros, siendo la octava estación en número de viajeros de la red de AVF española. Como en el caso del aeropuerto de Reus, la distribución anual de los viajeros en esta estación muestra, de forma clara, los efectos de la estacionalidad de la actividad turística. Durante los meses de julio y agosto alcanza el mayor volumen de viajeros, mientras que en la mayoría de estaciones de la red española de AVF durante estos meses se reduce el volumen de viajeros. Ello denota el peso especial del turismo estival en el comportamiento de la estación (Gutiérrez, 2009).

\section{Datos y metodología}

Los datos en los que se basa este estudio parten de una encuesta a viajeros de AVF realizada en la estación Camp de Tarragona entre el 13 de julio y el 24 de agosto de 2014. La muestra fue dimensionada con el objetivo de alcanzar un nivel de confianza del 95\%, con un margen de error del 5\%. A partir del volumen de viajeros de la estación durante el mismo período de 2013 (datos facilitados por Renfe) se cifró en 1.225 las encuestas a realizar. Estas se llevaron a cabo en la zona de embarque de la estación, durante los minutos previos a que los viajeros subieran a sus respectivos trenes. De acuerdo con el objetivo de este estudio, los resultados aquí explotados están basados en los pasajeros de nacionalidad española, no residentes en Cataluña, que regresaban a sus respectivos lugares de origen una vez finalizadas sus vacaciones en la Costa Dorada. Esto implica centrar el análisis en 519 pasajeros, un 42,4\% del total.

La encuesta consta de preguntas de carácter sociodemográfico (edad, sexo y lugar de origen); y también sobre las características del viaje y de la estancia, tales como tamaño del grupo y relación entre sus integrantes; si era su primera vez en el destino y, en caso que ya hubiesen estado en el destino, si era la primera vez que llegaban a través de la estación de AVF Camp de Tarragona; duración de la estancia; localidad de la estancia; tipo de alojamiento y tipo de traslado desde la estación hasta el destino final en la Costa Dorada. 
Figura 2. Regiones de origen de los pasajeros

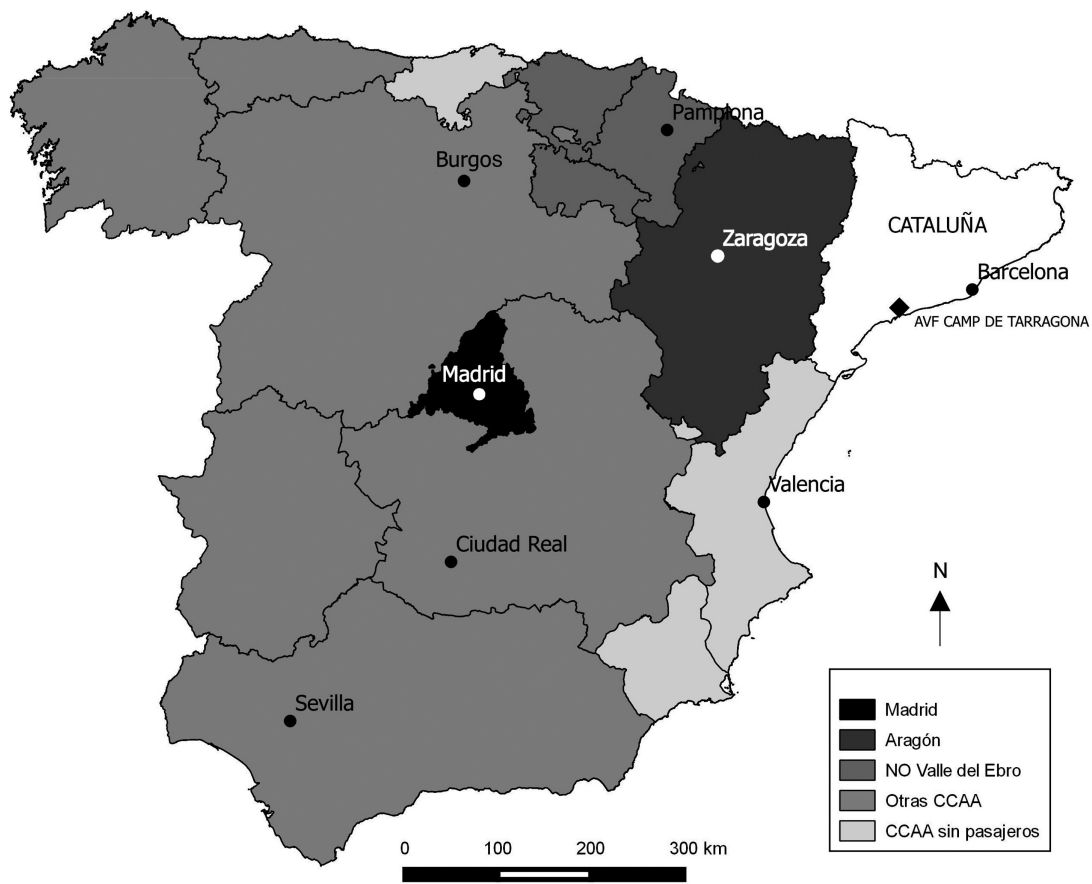

Fuente: elaboración propia.

En la figura 2 se muestra la distribución de los 519 turistas españoles encuestados según la comunidad autónoma de origen. En la tabla 1 se encuentra la distribución de estos turistas (números absolutos y relativos) agrupados en cuatro categorías. El grueso de este segmento de encuestados (dos de cada cinco) procede de Madrid. El trayecto entre las estaciones de Madrid-Puerta de Atocha y Camp de Tarragona tiene una duración de 2 h 30'. Casi una cuarta parte tienen su residencia en Aragón, la comunidad autónoma más cercana a Cataluña con conexión ferroviaria de alta velocidad. En este caso, el trayecto entre las estaciones de Zaragoza-Delicias y Camp de Tarragona tiene una duración de 1 h 20'. Así, alrededor de dos tercios de la muestra pertenecen únicamente a dos comunidades autónomas. Las otras dos categorías agrupan a diferentes comunidades autónomas. En la categoría III se encuentran los pasajeros con residencia en el País Vasco (34), Navarra (60) y La Rioja (7), un $19,5 \%$ del total. Se trata de las comunidades autónomas situadas en la zona noroccidental del valle del Ebro. A modo de ejemplo de las conexiones existentes con esta región, cabe apuntar que el trayecto entre las estaciones de Pamplona y Camp de Tarragona tiene una duración de 3 h 15'. Finalmente, la categoría IV (otras comunidades autónomas) engloba 83 pasajeros procedentes de seis comunidades autónomas: Andalucía (38), Asturias (3), Castilla-La 
Tabla 1. Distribución del conjunto de turistas españoles teniendo en cuenta su residencia

\begin{tabular}{llrc}
\hline & & N. & $\%$ \\
\hline I) & Aragón & 120 & $23,1 \%$ \\
II) & Madrid & 215 & $41,4 \%$ \\
III) & Noroeste del Valle del Ebro & 101 & $19,5 \%$ \\
IV) & Otras comunidades autónomas & 83 & $16,0 \%$ \\
\hline
\end{tabular}

Fuente: elaboración propia.

Mancha (16), Castilla y León (14), Extremadura (3) y Galicia (9). Se trata de comunidades autónomas que en su mayoría se encuentran mucho más alejadas de Cataluña. El trayecto entre las estaciones de Ciudad Real y Camp de Tarragona tiene una duración de 3h 10'; entre Sevilla-Santa Justa y Camp de Tarragona, 5 h; y entre las estaciones de Burgos y Camp de Tarragona, 5 h 30 '.

Los pasajeros de cada uno de los cuatro orígenes definidos se han distribuido teniendo en cuenta el resto de variables indicadas anteriormente, cosa que permitirá alcanzar el primer objetivo planteado. Se ha comprobado si las diferencias son estadísticamente significativas mediante la aplicación de la prueba del Chi Cuadrado $\left(\chi^{2}\right)$.

La encuesta también consta de otras dos preguntas que proporcionan los datos para aplicar el método de análisis causal y, de esta manera, alcanzar el segundo objetivo de este estudio.

- Pregunta causal 1. En una escala de 0 a 10, ¿̧hasta qué punto es cierto que has escogido la Costa Dorada como destino turístico debido a la existencia de la estación de AVF Camp de Tarragona? Siendo 10 totalmente cierto y 0 nada cierto.

- Pregunta causal 2. En una escala de 0 a 10, ¿hasta qué punto es cierto que hubieses escogido la Costa Dorada como destino turístico aunque no existiese la estación de AVF Camp de Tarragona? Siendo 10 totalmente cierto y 0 nada cierto.

A partir de una secuencia de dos preguntas formuladas de la manera expuesta, Young et al. (2005) desarrollaron un método probabilístico, que ellos denominan causal chain approach, por el cual es posible estudiar si cuando una persona es expuesta a cierta información sobre un producto o un servicio, ello puede tener un efecto en la decisión de consumir ese producto. En nuestro caso, la existencia de la estación (E) de AVF Camp de Tarragona puede propiciar que los turistas visiten (V) la Costa Dorada, por lo que puede identificarse como una posible causa de que la visita finalmente se produzca. No obstante, una única causa no tiene porqué ser ni necesaria ni suficiente para alcanzar un determinado objetivo (la llegada de turistas). La existencia de una estación de AVF no garantiza que un determinado territorio sea escogido como destino vacacional. Por otro lado, puede que esta infraestructura de transporte no sea necesaria para desplazarse hasta el destino en caso de que haya formas alterna- 
tivas disponibles para desplazarse. No obstante, la existencia de la estación de AVF Camp de Tarragona aumenta la probabilidad de visitar la Costa Dorada, ya que añade otro medio de transporte que permite acceder al destino. De esta manera, $\mathrm{P}(\mathrm{V} / \mathrm{E})$ en la ecuación 1 es la probabilidad de que una persona escoja como destino vacacional la Costa Dorada debido a la disponibilidad de una línea de alta velocidad ferroviaria con parada en la estación de AVF Camp de Tarragona, mientras que $\mathrm{P}(\mathrm{V} /-\mathrm{E})$ es la probabilidad que la visita se hubiese producido de todas maneras aunque la estación no hubiese existido (visita atribuible a otras causas ligadas al destino o a los propios turistas). La diferencia entre ellas es el incremento en la probabilidad $(\Delta \mathrm{P})$ de visitar el destino creado por la existencia de la estación de alta velocidad ferroviaria.

$$
\Delta \mathrm{P}=\mathrm{P}(\mathrm{V} \mid \mathrm{E})-\mathrm{P}(\mathrm{V} \mid-\mathrm{E})(\text { Ecuación } 1)
$$

La primera de las dos preguntas causales indicadas anteriormente está diseñada para obtener la probabilidad de que la visita al destino sea debida a la existencia de la estación de AVF ( $\mathrm{k}_{\mathrm{i}}$ en la ecuación 2). La segunda pregunta se refiere a la probabilidad de que, sin la existencia de la estación de AVF Camp de Tarragona, los turistas hubieran escogido la Costa Dorada como destino turístico de todos modos $\left(1-\mathrm{c}_{\mathrm{i}}\right)$. Entonces, $\mathrm{c}_{\mathrm{i}}$ es la probabilidad de que sin la estación de AVF no se hubiese producido la visita al destino.

$$
\Delta \mathrm{p}_{\mathrm{i}}=\mathrm{k}_{\mathrm{i}} * \mathrm{c}_{\mathrm{i}} \text { (Ecuación 2) }
$$

Los encuestados han de responder valores entre 0 y 10 tanto a la primera como a la segunda pregunta causal. Ambas variables de la ecuación $2\left(\mathrm{k}_{\mathrm{i}} \mathrm{y}_{\mathrm{i}}\right)$ evalúan el efecto ejercido por la disponibilidad de la estación de AVF en la elección del destino turístico, pero de diferente manera. El efecto neto se expresa en base a su producto $\left(\Delta \mathrm{p}_{\mathrm{i}}\right)$, tal como indican Fernandez Young and Young (2008). Aunque existe un amplio abanico de combinaciones posibles, es de esperar que aquellos pasajeros que a la primera pregunta respondan un número cercano a 0 respondan un número cercano a 10 a la segunda y viceversa. Para el cálculo de $\Delta \mathrm{p}_{\mathrm{i}}$, los valores de $\mathrm{k}_{\mathrm{i}} \mathrm{y}_{\mathrm{i}}$ se transforman a una escala entre 0 y 1 .

Los extremos de la gama de respuestas son, por un lado, aquellos pasajeros que manifiestan que la existencia de la estación de AVF Camp de Tarragona no ha tenido influencia alguna en la decisión de haber escogido la Costa Dorada como destino turístico y que la hubieran escogido de todos modos aunque la estación no hubiese existido ( $\mathrm{P} 1=0$ y $\mathrm{P} 2=10$ ). Por consiguiente, los valores de $\mathrm{k}_{\mathrm{i}} \mathrm{y}$ de $\mathrm{c}_{\mathrm{i}}$ serían igual a 0 . En el extremo opuesto se encontrarían los pasajeros que manifiestan que el hecho de haber escogido la Costa Dorada como destino turístico es debido totalmente a la existencia de la estación de AVF Camp de Tarragona y que no hubieran visitado la Costa Dorada en caso de no haber existido la estación $(\mathrm{P} 1=10$ y P2 $=0)$. Por consiguiente, los valores de $\mathrm{k}_{\mathrm{i}} \mathrm{y}$ de $\mathrm{c}_{\mathrm{i}}$ serían igual a 10. En el primer grupo, la estación de alta velocidad ferroviaria no influye en que se produzca la visita al destino, mientras que en el segundo 
grupo únicamente la existencia de la estación de AVF Camp de Tarragona explica la visita a la Costa Dorada. En consecuencia, $\Delta \mathrm{p}_{\mathrm{i}}$ (ecuación 2) es el porcentaje o fracción de la visita de cada uno de los integrantes de la muestra atribuible a la existencia de la estación de AVF Camp de Tarragona. Esto permite medir la contribución del conjunto de la muestra, o de segmentos seleccionados, en base a la suma de las fracciones individuales ( $\Delta \mathrm{p}$ en ecuación 3$)$. Un cálculo más preciso que clasificar a los turistas en base a una respuesta de tipo binario (p. ej. Sí/No), donde las fracciones individuales se perderían.

$$
\Delta \mathrm{p}=\sum_{\mathrm{i}=1}^{\mathrm{N}}\left(\mathrm{k}_{\mathrm{i}} * \mathrm{c}_{\mathrm{i}}\right) / \mathrm{N}(\text { Ecuación 3) }
$$

Esta metodología se ha aplicado a los cuatro segmentos de la muestra seleccionados: usuarios españoles de la estación de AVF Camp de Tarragona que han estado de vacaciones en la Costa Dorada y que regresan a sus residencias situadas en Aragón, Madrid, noroeste del Valle del Ebro y otras comunidades autónomas.

\section{Resultados}

\subsection{Caracterización de los turistas según su procedencia}

Las tablas 2 y 3 muestran las características sociodemográficas y de la estancia en la Costa Dorada de los pasajeros incluidos en cada uno de los cuatro segmentos definidos en base a su procedencia. También se indica si las diferencias entre estos cuatro segmentos son estadísticamente significativas. Hay una mayor presencia de mujeres en tres de los cuatro grupos. Esta situación es más notable en el caso de los turistas procedentes de Aragón (seis de cada diez). El promedio de edad es muy similar en todos los casos, desde los 40 años en Aragón a los 43 en las comunidades situadas en el noroeste del Valle del Ebro. En cambio, sí que aparecen diferencias significativas en base al establecimiento de grupos de edad. La mayor frecuencia se encuentra en el grupo de edad de 41-60 años entre los pasajeros procedentes del noroeste del Valle del Ebro y de «otras comunidades autónomas», mientras que hay una mayor presencia de aquellos con edades comprendidas entre los 26 y 40 años entre los pasajeros con residencia en Aragón y, especialmente, en Madrid (47,4\%). Cabe destacar el contraste entre los pasajeros más jóvenes (18-25 años) procedentes de Madrid y de "otras comunidades autónomas». Mientras que en el primer caso representan poco más del 10\%, en el segundo superan el $20 \%$.

El promedio de edad aumenta cuanto mayor es la distancia entre el origen y el destino y, por consiguiente, aumenta el precio del billete de tren. Sin embargo, este es un patrón que no se ajusta en el caso de los pasajeros procedentes de "otras comunidades autónomas». El promedio de edad en este grupo es el mismo que en el caso de los procedentes de Madrid (41 años). No obstante, cabe recordar que en esta categoría se incluyen turistas procedentes de diferentes regiones de España (p. ej. Andalucía, 44 años; Castilla-La Mancha, 34 años). Los valores son menos dispares en relación con el grupo de mayor 
Tabla 2. Características sociodemográficas y de grupo

\begin{tabular}{|c|c|c|c|c|}
\hline & Aragón & Madrid & $\begin{array}{l}\text { Noroeste del } \\
\text { Valle del Ebro' }\end{array}$ & Otras CCAA \\
\hline \multicolumn{5}{|c|}{$\begin{array}{l}\text { Sexo } \chi^{2}(3 \mathrm{gl})=1,908 \\
\text { estadísticamente no significativo }\end{array}$} \\
\hline Mujeres & $72(60,0 \%)$ & $118(55,1 \%)$ & $58(57,4 \%)$ & $41(49,4 \%)$ \\
\hline Hombre & $48(40,0 \%)$ & $96(44,9 \%)$ & $43(42,6 \%)$ & $42(50,6 \%)$ \\
\hline \multicolumn{5}{|c|}{$\begin{array}{l}\text { Edad } \\
\chi^{2}(9 \mathrm{gl})=18,787 ; p<0,05\end{array}$} \\
\hline 18-25 años & $22(18,3 \%)$ & $22(10,3 \%)$ & 15 (14,9\%) & $18(21,7 \%)$ \\
\hline 26-40 años & $46(38,3 \%)$ & $101(47,4 \%)$ & $30(29,7 \%)$ & $23(27,7 \%)$ \\
\hline 41-60 años & $41(34,2 \%)$ & $68(31,9 \%)$ & $42(41,6 \%)$ & $32(38,6 \%)$ \\
\hline > 60 años & $11(9,2 \%)$ & $22(10,4 \%)$ & $14(13,8 \%)$ & $10(12,0 \%)$ \\
\hline Promedio & 40 años & 41 años & 43 años & 41 años \\
\hline \multicolumn{5}{|c|}{$\begin{array}{l}\text { Tamaño grupo } \\
\chi^{2}(9 \mathrm{gl})=20,377 ; p<0,05\end{array}$} \\
\hline 1 persona & $72(60,0 \%)$ & $107(49,8 \%)$ & $40(39,6 \%)$ & $34(41,0 \%)$ \\
\hline 2 personas & $24(20,0 \%)$ & $49(22,8 \%)$ & $29(28,7 \%)$ & $18(21,7 \%)$ \\
\hline 3 personas & $14(11,7 \%)$ & $28(13,0 \%)$ & $17(16,8 \%)$ & $9(10,8 \%)$ \\
\hline$>3$ personas & $10(8,3 \%)$ & $31(14,4 \%)$ & $15(14,9 \%)$ & $22(26,5 \%)$ \\
\hline \multicolumn{5}{|c|}{$\begin{array}{l}\text { Estructura } \chi^{2}(9 \mathrm{gl})=12,763 \\
\text { estadísticamente no significativo }\end{array}$} \\
\hline 1 persona & $72(60,0 \%)$ & $107(49,8 \%)$ & $40(39,6 \%)$ & $34(41,0 \%)$ \\
\hline Familia-hijos & $17(14,2 \%)$ & $41(19,1 \%)$ & $30(29,7 \%)$ & $23(27,7 \%)$ \\
\hline Familiares adultos & $19(15,8 \%)$ & $46(21,4 \%)$ & $19(18,8 \%)$ & $18(21,7 \%)$ \\
\hline Amigos adultos & $12(10,0 \%)$ & $21(9,8 \%)$ & $12(11,9 \%)$ & $8(9,6 \%)$ \\
\hline
\end{tabular}

1. Incluye La Rioja, Navarra y el País Vasco.

Fuente: elaboración propia.

edad (> 60 años), oscilando entre el 9,2\% entre los procedentes de Aragón y el $13,8 \%$ de los del noroeste del Valle del Ebro.

Teniendo en cuenta el tamaño del grupo, en todos los casos el más numeroso es el de un turista que realizó el viaje solo. No obstante, mientras que entre los procedentes del noroeste del Valle del Ebro son un 39,6\%, la cifra alcanza el $60 \%$ entre los pasajeros de Aragón. En estos dos grupos se produce una disminución de la presencia de pasajeros a medida que aumenta el tamaño del grupo, situación que no se produce en el caso de aquellos procedentes de Madrid y, especialmente, entre los procedentes de "otras comunidades autónomas». En este segundo caso, más de una cuarta parte del total manifestó formar un grupo de cuatro personas o más. Una cifra que no alcanza el $10 \%$ entre los turistas procedentes de Aragón. La presencia de grupos más numerosos aumenta cuanto mayor es la distancia entre el origen y el destino turístico. La relación entre los pasajeros que no viajaron solos (grupos de dos o más personas) muestra que predominan aquellos formados por familiares adultos en el 
Tabla 3. Características de la estancia

\begin{tabular}{|c|c|c|c|c|}
\hline & Aragón & Madrid & $\begin{array}{l}\text { Noroeste del } \\
\text { Valle del Ebro' }\end{array}$ & Otras CCAA \\
\hline \multicolumn{5}{|c|}{$\begin{array}{l}1^{\text {a }} \text { vez en el destino } \\
\chi^{2}(3 \mathrm{gl})=17,132 ; p<0,001\end{array}$} \\
\hline Sí & $5(4,2 \%)$ & $31(14,4 \%)$ & $10(9,9 \%)$ & $19(22,9 \%)$ \\
\hline No & $115(95,8 \%)$ & $184(85,6 \%)$ & $91(90,1 \%)$ & $64(77,1 \%)$ \\
\hline \multicolumn{5}{|c|}{$\begin{array}{l}1^{\text {a vez estación }} \\
\chi^{2}(3 \mathrm{gl})=21,784 ; p<0,001\end{array}$} \\
\hline Sí & $42(36,8 \%)$ & $42(22,8 \%)$ & $51(56,0 \%)$ & $32(50,0 \%)$ \\
\hline No & $73(63,2 \%)$ & $142(77,2 \%)$ & $40(44,0 \%)$ & $32(50,0 \%)$ \\
\hline \multicolumn{5}{|c|}{$\begin{array}{l}\text { Transporte estación } \\
\chi^{2}(6 \mathrm{gl})=31,592 ; p<0,001\end{array}$} \\
\hline Vehículo privado & $83(69,7 \%)$ & $151(72,9 \%)$ & $45(45,0 \%)$ & $47(59,5 \%)$ \\
\hline Autobús de línea & $23(19,4 \%)$ & $23(11,2 \%)$ & $22(22,0 \%)$ & $17(21,5 \%)$ \\
\hline Taxi & $13(10,9 \%)$ & $33(15,9 \%)$ & $33(33,0 \%)$ & $15(19,0 \%)$ \\
\hline \multicolumn{5}{|c|}{$\begin{array}{l}\text { Localidad } \chi^{2}(12 \mathrm{gl})=19,193 ; \\
\text { estadísticamente no significativo }\end{array}$} \\
\hline Tarragona ciudad & $15(12,5 \%)$ & $27(12,6 \%)$ & 7 (6,9\%) & $17(20,5 \%)$ \\
\hline Cambrils & $24(20,1 \%)$ & $41(19,1 \%)$ & $24(23,8 \%)$ & $9(10,8 \%)$ \\
\hline La Pineda & $13(10,8 \%)$ & $11(5,1 \%)$ & $10(9,9 \%)$ & $5(6,1 \%)$ \\
\hline Salou & $25(20,8 \%)$ & $57(26,5 \%)$ & $31(30,7 \%)$ & $25(30,1 \%)$ \\
\hline Resto Costa Dorada & $43(35,8 \%)$ & $79(36,7 \%)$ & $29(28,7 \%)$ & $27(32,5 \%)$ \\
\hline \multicolumn{5}{|c|}{$\begin{array}{l}\text { Alojamiento } \\
\chi^{2}(9 \mathrm{gl})=48,771 ; p<0,001\end{array}$} \\
\hline Propio & $34(28,3 \%)$ & $44(20,6 \%)$ & $18(17,8 \%)$ & $6(7,3 \%)$ \\
\hline Familiares/amigos & $48(40,0 \%)$ & $100(46,7 \%)$ & $20(19,8 \%)$ & $43(51,8 \%)$ \\
\hline Hotel & $20(16,7 \%)$ & $50(23,4 \%)$ & $38(37,6 \%)$ & $24(28,9 \%)$ \\
\hline Apartamento/Camping & $18(15,0 \%)$ & $20(9,3 \%)$ & $25(24,8 \%)$ & $10(12,0 \%)$ \\
\hline \multicolumn{5}{|c|}{$\begin{array}{l}\text { Pernoctaciones } \\
\chi^{2}(3 \mathrm{gl})=38,370 ; p<0,001\end{array}$} \\
\hline$\leq 3$ noches & $50(42,0 \%)$ & $60(28,2 \%)$ & $10(10,0 \%)$ & $10(12,2 \%)$ \\
\hline$>3$ noches & $69(58,0 \%)$ & $153(71,8 \%)$ & $90(90,0 \%)$ & $72(87,8 \%)$ \\
\hline Promedio & 7 noches & 8 noches & 9 noches & 12 noches \\
\hline
\end{tabular}

1. Incluye La Rioja, Navarra y el País Vasco.

Fuente: elaboración propia.

caso de aragoneses y madrileños, mientras que en el caso de los procedentes del noroeste del Valle del Ebro y de "otras comunidades autónomas» son familias con hijos. Finalmente, en todos los casos la estructura menos habitual es la de un grupo formado por amigos adultos (alrededor del 10\%).

Una cuestión importante es saber si los turistas que llegan a la Costa Dorada a través de la estación de AVF Camp de Tarragona ya han estado de vacaciones en el destino y, en caso afirmativo, si también habían utilizado la estación de AVF Camp de Tarragona. La tabla 3 muestra que, independientemente del 
lugar de origen de los turistas, en la gran mayoría de casos no era la primera vez que escogían la Costa Dorada como destino vacacional. Ahora bien, a pesar de ello, las diferencias son estadísticamente significativas. Poco más de un 4\% de los turistas procedentes de Aragón no habían estado antes en la Costa Dorada, mientras que la cifra de estos «nuevos» turistas alcanza un $22,9 \%$ en el caso de los procedentes de "otras comunidades autónomas». De nuevo la distancia entre el lugar de origen y el destino juega un papel importante. Por su parte, la mayoría de los turistas procedentes de Aragón y de Madrid que ya habían visitado en otras ocasiones la Costa Dorada también habían utilizado la estación de AVF Camp de Tarragona. En cambio, son la mitad en el caso de los procedentes de "otras comunidades autónomas» y menos de la mitad entre los que tienen su residencia en las comunidades autónomas situadas en el noroeste del Valle del Ebro.

Los datos evidencian un cambio en la modalidad de transporte de los turistas que en el momento de hacer la encuesta viajan en AVF. Este es un resultado relevante. En efecto, más de la mitad de los turistas procedentes de las regiones situadas en el noroeste del Valle del Ebro que ya habían estado en el destino en otras ocasiones, era la primera vez que llegaban a la Costa Dorada a través de la estación de AVF Camp de Tarragona. En el caso de los procedentes de Aragón, estos son más de un tercio, cosa que denota una substitución del vehículo privado por la AVF (Zaragoza-Delicias y Camp de Tarragona: $1 \mathrm{~h}$ 20 '). En cambio, menos de una cuarta parte del total de los procedentes de Madrid manifestó que era la primera vez que llegaban al destino a través de la estación de AVF Camp de Tarragona. Si comparamos las dos procedencias con un mayor número de turistas (Madrid y Aragón), la distancia con la Costa Dorada vuelve a jugar un papel destacado. La estación de AVF Camp de Tarragona atrae una menor cantidad (relativa y números absolutos) de nuevos turistas procedentes de Aragón y más de Madrid. Por el contrario, Aragón es la comunidad donde un mayor volumen de turistas (en términos absolutos y relativos) que anteriormente ya habían visitado el destino han realizado un cambio de modo de transporte a favor de la AVF.

La estación de AVF Camp de Tarragona es periférica en relación con la ciudad de Tarragona y las principales localidades turísticas de la Costa Dorada. Entre las estaciones periféricas, la accesibilidad a y desde el destino del viaje es un factor clave, ya que repercute de forma directa sobre el tiempo y calidad del desplazamiento total (Faccinetti-Mannone, 2005). En este sentido, es interesante conocer qué medio de transporte utilizan los usuarios de la estación de AVF Camp de Tarragona para desplazarse desde la estación hasta su destino vacacional. Existen diferentes líneas de autobús que conectan la estación con las ciudades de Tarragona y Reus, el aeropuerto de Reus y los núcleos del litoral de Salou, Cambrils, Vila-seca y La Pineda. Su frecuencia, especialmente con las localidades de la Costa Dorada Central y el aeropuerto, es mayor en verano. También hay servicio de taxi. No obstante, el principal modo de transporte para acceder desde la estación al destino final es el vehículo privado; predominan claramente los pasajeros que fueron recogidos por amigos o familiares. 
El porcentaje oscila entre el $45 \%$ de los turistas procedentes del noroeste del Valle del Ebro y el 72,9\% de los de Madrid. El autobús de línea es el segundo modo de transporte más habitual entre los residentes en Aragón y de «otras comunidades autónomas». La segunda posición es ocupada por el taxi entre los residentes en Madrid y, especialmente, entre los del noroeste del Valle del Ebro (33\%). Así, la primera característica definitoria de las pautas de movilidad de los viajeros de esta estación es el uso del vehículo privado, siendo un familiar o amigo el que los recoge en la estación. Existe una clara preponderancia de pasajeros que no tienen que preocuparse por la disponibilidad de servicio de taxi o de autobús de línea en la estación, puesto que ya saben que les estarán esperando para trasladarlos al destino. Unos resultados estrechamente ligados con los obtenidos en el tamaño del grupo (predominio de personas que viajan solas) y, tal como se verá más adelante, con el tipo de alojamiento en el destino. Esta distribución modal cambia de manera significativa en estaciones centrales, donde el uso de taxi, transporte público y el acceso a pie son fórmulas más utilizadas (Burckhart et al., 2008).

Salou es la localidad de la Costa Dorada que recibe a más turistas entre los llegados a la estación de AVF Camp de Tarragona (hasta un 30\% de los procedentes del noroeste del Valle del Ebro y de «otras comunidades autónomas»). Entre los aragoneses se da el valor más bajo (uno de cada cinco). Alrededor de la mitad de los turistas procedentes de Aragón, Madrid y «otras comunidades autónomas» escogieron la Costa Dorada Central (Salou-Cambrils-La Pineda), cifra que asciende hasta los dos tercios en el caso de los procedentes del noroeste del Valle del Ebro. La ciudad de Tarragona es escogida, en término relativos, en mayor medida por los turistas procedentes de «otras comunidades autónomas» (20,5\%). No obstante, en tres de los cuatros segmentos analizados el grupo más numeroso es el de aquellos que estuvieron en «otras localidades de la Costa Dorada».

En relación con el tipo de alojamiento, la opción mayoritaria son las viviendas de amigos o familiares, con la excepción de los procedentes del noroeste del Valle del Ebro (un 37,6\% se alojaron en hotel). El hotel es la segunda opción principal entre los llegados de Madrid y «otras comunidades autónomas». Mientras que entre los procedentes de Aragón lo es la segunda residencia en el destino. Todo ello implica que menos de un tercio del total de turistas procedentes de Aragón y de Madrid estuvieron alojados en establecimientos turísticos reglados, ya sean hoteles, apartamentos o campings. En el otro extremo se encuentran los procedentes del noroeste del Valle del Ebro con un $62,4 \%$. A mayor distancia del origen, mayor peso del hotel; mientras que, a menor distancia, mayor peso del alojamiento en segunda residencia de familiares, amigos o propia.

Finalmente, las diferencias son evidentes teniendo en cuenta la duración de la estancia, diferenciando entre aquellos que estuvieron hasta tres noches o más. El primer grupo son considerados como turistas de corta estancia por parte de la OMT (2010). El peso relativo de los que estuvieron como máximo tres noches oscila entre el $42 \%$ de los procedentes de Aragón y el 10\% de los del 
noroeste del Valle del Ebro. De nuevo la distancia entre el origen y el destino juega un papel importante. A mayor distancia, mayor es el peso relativo de los que estuvieron más de tres noches y también el promedio de pernoctaciones: siete en un turista aragonés y doce en uno procedente de «otras comunidades autónomas".

\subsection{Incidencia de la AVF en la elección del destino según el origen de los turistas}

El análisis anterior destaca que la AVF no está generando una importante llegada de nuevos turistas a la Costa Dorada. En cambio, se observa un cierto cambio en el modo de transporte para desplazarse hasta un destino ya visitado en anteriores ocasiones. No obstante, es posible que una parte de los pasajeros que, por primera vez, han escogido la Costa Dorada como destino lo hubiesen hecho de todos modos aunque no hubiera existido el servicio de AVF. Y, por otro lado, también es posible que una parte de los pasajeros que repiten estancia en la Costa Dorada no la hubieran escogido en caso de que no existiese la estación de AVF Camp de Tarragona. Teniendo en cuenta estas premisas, a continuación se analiza el incremento en la probabilidad de visitar la Costa Dorada, debido a la existencia de la estación de AVF.

La tabla 4 muestra la distribución de los valores extremos de las dos preguntas causales planteadas a los pasajeros teniendo en cuenta los cuatro grupos determinados en base a su procedencia. El peso relativo de los pasajeros que respondieron 0 a la primera pregunta causal, es decir, la estación de tren no ha tenido nada que ver en la decisión de realizar las vacaciones en la Costa Dorada, oscila entre el 26,1\% de los procedentes de Madrid y el 40,6\% de los procedentes del noroeste del Valle del Ebro. Por el contrario, los pasajeros que dieron como respuesta un 10, o lo que es lo mismo, manifestaron que es totalmente cierto que la decisión de ir a la Costa Dorada fue debida a la existencia de la estación de AVF Camp de Tarragona, tienen un peso relativo mucho menor. En este segundo caso, como es de esperar, las posiciones se invierten. De esta manera el porcentaje oscila entre el 1,4\% de los procedentes de Madrid y el 5\% de los procedentes del noroeste del Valle del Ebro.

Tabla 4. Frecuencia de los valores extremos en las preguntas causales

\begin{tabular}{lrrrr}
\hline & Aragón & Madrid & $\begin{array}{c}\text { Noroeste del } \\
\text { Valle del Ebro }\end{array}$ & Otras CCAA \\
\hline P1 $=0$ & $31,7 \%$ & $26,1 \%$ & $40,6 \%$ & $33,7 \%$ \\
P1 $=10$ & $1,7 \%$ & $1,4 \%$ & $5,0 \%$ & $3,6 \%$ \\
P2 $=0$ & $3,3 \%$ & $7,9 \%$ & $6,9 \%$ & $7,2 \%$ \\
P2 $=10$ & $40,8 \%$ & $35,8 \%$ & $50,5 \%$ & $45,8 \%$ \\
P1 $=0$ y P2 $=10$ & $25,0 \%$ & $24,2 \%$ & $35,6 \%$ & $28,9 \%$ \\
P1 $=10$ y P2 =0 & $0,8 \%$ & $1,4 \%$ & $3,0 \%$ & $2,4 \%$ \\
\hline
\end{tabular}

1. Incluye La Rioja, Navarra y el País Vasco.

Fuente: elaboración propia. 
Las respuestas a la segunda pregunta causal muestran, en todos los casos, una importante presencia de pasajeros que manifestaron que era totalmente cierto $(\mathrm{P} 2=10)$ que hubiesen visitado la Costa Dorada aunque la estación de AVF Camp de Tarragona no hubiese existido. En los extremos encontramos las dos mismas regiones que anteriormente: más de la mitad de los procedentes del noroeste del Valle del Ebro y poco más de un tercio de los procedentes de Madrid. Por su parte, hubo pocos pasajeros, independientemente de su origen, que indicasen que fuera totalmente seguro que, en caso de no existir la estación de AVF, no hubiesen escogido la Costa Dorada como destino $(\mathrm{P} 2=0)$.

Teniendo en cuenta la combinación de los valores extremos de cada una de las dos preguntas, podemos afirmar que únicamente un $0,8 \%$ de pasajeros de Aragón y un 1,4\% de los de Madrid pueden ser considerados «turistas determinados por la AVF». Estos son aquellos para los que la estancia en la Costa Dorada fue única y exclusivamente debida a la existencia de la estación de AVF Camp de Tarragona. Estos pasajeros respondieron que era totalmente cierto que la visita era debida a la existencia de la estación $(\mathrm{P} 1=10$, luego $\mathrm{k}_{\mathrm{i}}=1$ ) y que de ninguna manera se hubiese producido en el caso que la estación no hubiese existido ( $\mathrm{P} 2=0$, luego $\left.\mathrm{c}_{\mathrm{i}}=1\right)$. En estos casos el incremento en la probabilidad de visita a la Costa Dorada debido a la existencia de la estación de AVF Camp de Tarragona fue del 100\% $\left(\Delta \mathrm{p}_{\mathrm{i}}=1\right)$. En el otro extremo se encuentran los que indicaron que la estación no había tenido ninguna influencia en la toma de decisión ( $\mathrm{P} 1=0$; luego $\left.\mathrm{k}_{\mathrm{i}}=0\right) \mathrm{y}$, a su vez, que en caso que la estación no hubiese existido hubiesen viajado de todos modos hasta la Costa Dorada por otros medios $\left(\mathrm{P} 2=10\right.$, luego $\left.\mathrm{c}_{\mathrm{i}}=0\right)$. El incremento en la probabilidad de visita creado por la estación de AVF Camp de Tarragona es 0 . Se trata de pasajeros que pueden denominarse como «turistas determinados por el destino». En esta situación encontramos una cuarta parte de los procedentes de Aragón y Madrid y más de un tercio de los del noroeste del Valle del Ebro.

La distribución de los 519 pasajeros teniendo en cuenta la combinación de las respuestas dadas a las dos preguntas causales se muestra en las figuras 3 (Aragón - Madrid) y 4 (NO del Valle del Ebro - otras comunidades autónomas). El incremento en la probabilidad de visita al destino creado por la estación también fue del $0 \%$ en aquellos otros turistas que respondieron 0 a la pregunta 1 y un valor diferente de 10 a la pregunta 2 ; así como también los que respondieron 10 a la pregunta 2 y un valor diferente de 0 a la pregunta 1 . El porcentaje de turistas condicionados en mayor o menor medida (desde 0,01 hasta 1 - donde 1 es un incremento de la probabilidad del 100\%) por la existencia de la estación de AVF Camp de Tarragona oscila entre el 62,5\%, que experimenta algún tipo de influencia, entre los procedentes de Madrid y el 44,5\% entre los procedentes del noroeste del Valle del Ebro.

A partir de los resultados individuales fruto de la combinación de las dos preguntas causales podemos cuantificar, para cada uno de los cuatro segmentos de pasajeros, la influencia directa de la estación en la elección del destino, la probabilidad que la visita se hubiese producido aunque la estación no hubiera 
Figura 3. Incremento individual en la probabilidad de visitar la Costa Dorada debido a la estación de AVF Camp de Tarragona: Aragón (arriba) y Madrid (abajo)
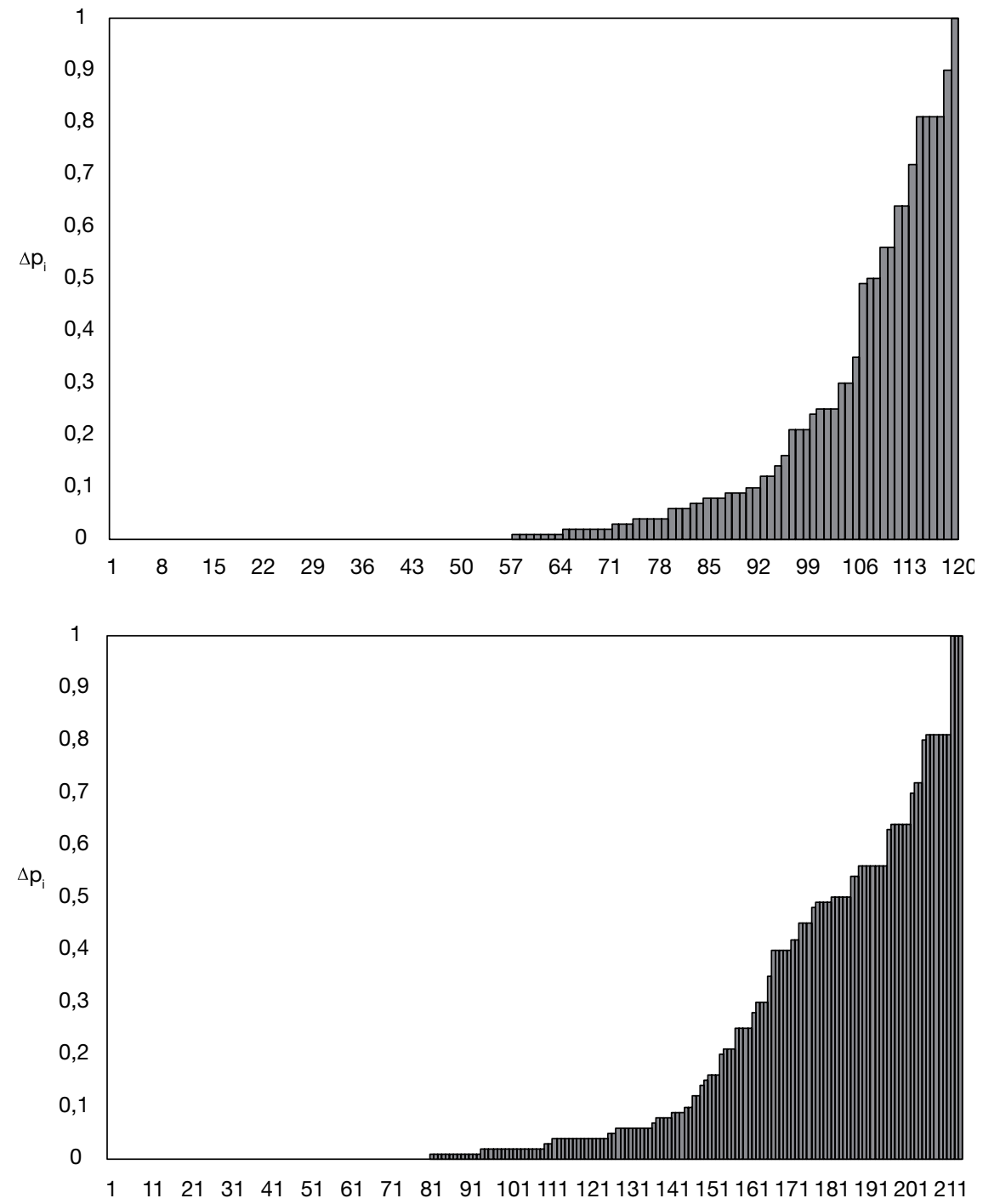

Fuente: elaboración propia. 
Figura 4. Incremento individual en la probabilidad de visitar la Costa Dorada debido a la estación de AVF Camp de Tarragona: noroeste del Valle del Ebro (arriba) y otras comunidades autónomas (abajo)
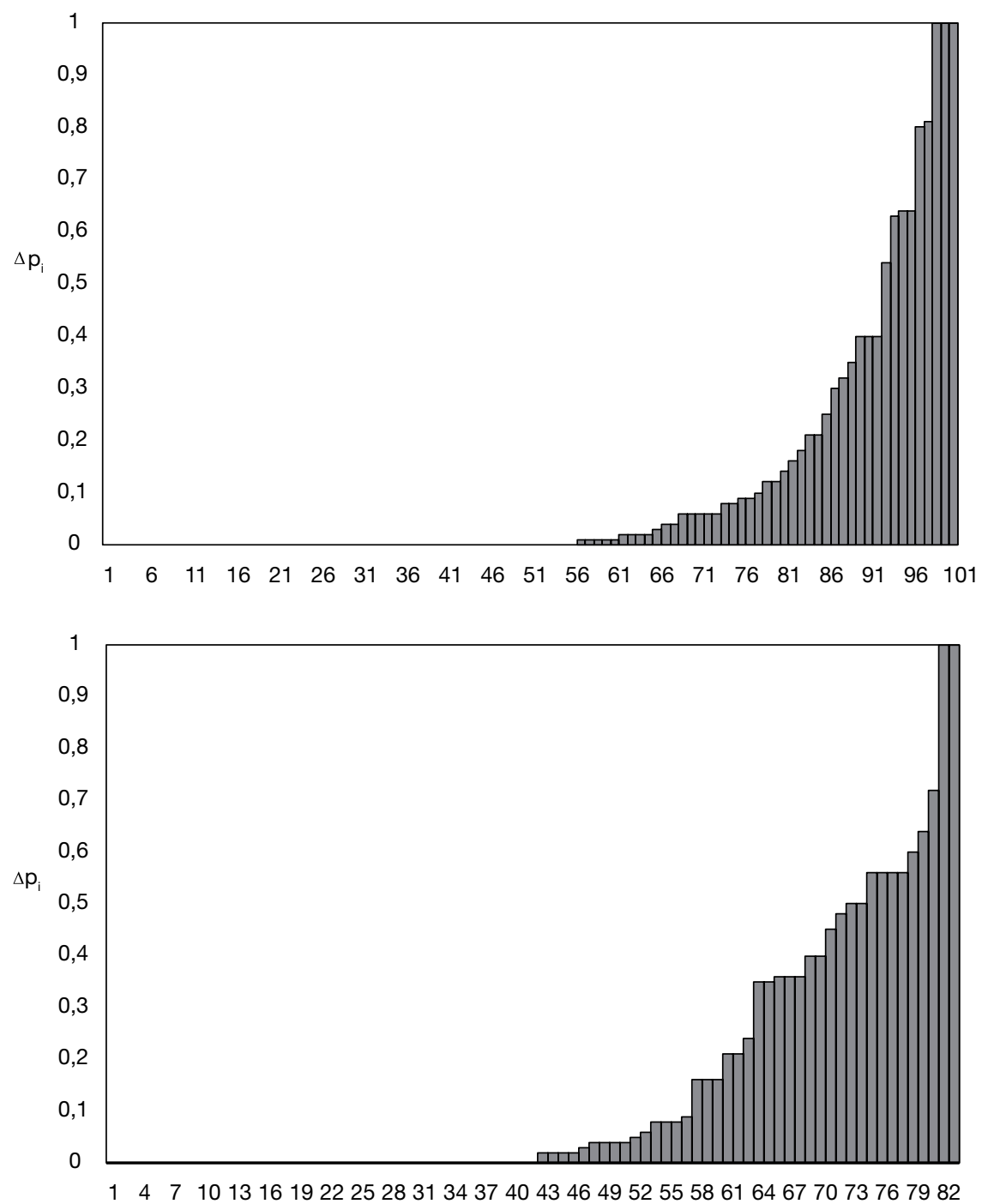

Fuente: elaboración propia. 
Tabla 5. AVF y elección del destino turístico según el origen de los pasajeros

\begin{tabular}{lcccc}
\hline & Aragón & Madrid & $\begin{array}{c}\text { Noroeste del } \\
\text { Valle del Ebro }\end{array}$ & Otras CCAA \\
\hline $\mathrm{k}$ & 0,308 & 0,361 & 0,316 & 0,381 \\
$1-\mathrm{c}$ & 0,801 & 0,744 & 0,817 & 0,765 \\
$\Delta \mathrm{p}$ & 0,120 & 0,166 & 0,115 & 0,151 \\
\hline
\end{tabular}

1. Incluye La Rioja, Navarra y el País Vasco.

Fuente: elaboración propia.

existido y el incremento en la probabilidad de visitar la Costa Dorada creado por la existencia de la estación de AVF Camp de Tarragona (tabla 5).

La influencia $(\mathrm{k})$ es moderada en todos los casos. No obstante, la estación ha ejercido una mayor influencia entre aquellos pasajeros procedentes de «otras comunidades autónomas» $(38,1 \%)$, entre las que se encuentran aquellas más distantes como Andalucía. Un valor similar al obtenido en el caso de los de Madrid (36,1\%). Los provenientes de Aragón, la región más cercana, son, con un $30,8 \%$, los pasajeros menos influenciados por la existencia de la estación de AVF en el momento de escoger el destino donde pasar las vacaciones. Por otro lado, el destino (1-c) ejerce una más que notable influencia (independientemente de la existencia de la estación) en el hecho de haber sido elegido: un $81,7 \%$ entre los procedentes del noroeste del Valle del Ebro. Un valor muy parejo al obtenido en el caso de Aragón. Por su parte no alcanza un 75\% entre los procedentes de Madrid, cifra ligeramente inferior a la de aquellos pasajeros de «otras comunidades autónomas» (76,5\%). Unos resultados que, en todos los casos, denotan un elevado grado de fidelidad al destino por parte de los pasajeros, aunque mayor entre los más próximos al destino.

En consecuencia, el incremento en la probabilidad de visitar la Costa Dorada $(\Delta \mathrm{p})$ creado por la existencia de la estación de AVF Camp de Tarragona es más elevado entre los turistas procedentes de Madrid (16,6\%) y de «otras comunidades autónomas» $(15,1 \%)$. En este último grupo hay que destacar que alcanza un $21 \%$ entre aquellos procedentes de Andalucía. No obstante, apenas representan un 7\% del total de turistas que llegan en AVF a la Costa Dorada. En el otro extremo se encuentran los procedentes de Aragón (12\%) y los del noroeste del Valle del Ebro (11,5\%).

\section{Conclusiones}

En este estudio hemos analizado las características (sociodemográficas, del viaje y de la estancia) de los turistas españoles que, teniendo en cuenta su región de origen, estuvieron de vacaciones en un destino turístico de litoral, como es la Costa Dorada en Cataluña, y que utilizaron como medio de transporte la alta velocidad ferroviaria. Los turistas españoles suponen un $57 \%$ del total de los llegados a la Costa Dorada. Entre estos, un 4\% lo hace a través de alta velocidad ferroviaria. Se trata, por tanto, de un colectivo con un peso 
reducido entre el total de turistas llegados a este destino. Sin embargo, el interés de su estudio reside en la falta de antecedentes sobre el análisis de la incidencia de la alta velocidad ferroviaria en la elección de un determinado destino turístico.

La primera conclusión de nuestro trabajo es que la mayor o menor distancia entre el lugar de origen y el destino turístico condiciona el perfil de los turistas usuarios de la AVF, existiendo diferencias significativas. Entre los que residen más cerca del destino hay una mayor presencia relativa de aquellos que viajan solos y que han realizado una estancia de como máximo tres noches. Por contra, entre los pasajeros procedentes de regiones más alejadas hay una mayor presencia relativa de aquellos que viajan en grupos de más de tres personas, alojados en un hotel y que realizan una estancia superior a tres noches.

En segundo lugar, los resultados demuestran que la incorporación de nuevos turistas (que visitan por primera vez la Costa Dorada y viajan en AVF) aumenta cuanto mayor es la distancia entre el destino y el lugar de origen. Este resultado está en gran parte justificado por el hecho de que la conexión entre la estación de AFV Camp de Tarragona con, por ejemplo, las existentes en las regiones más meridionales de España, es mucho más reciente que con Zaragoza Delicias o Madrid Atocha y, por tanto, evidencia el papel que juega el transporte en el desarrollo turístico. No obstante, los turistas procedentes de las regiones más alejadas representan un porcentaje bajo del total y, por otro lado, en todos los casos hay una mayor presencia de turistas que ya han visitado anteriormente la Costa Dorada. De este modo, una tercera conclusión es que, más que incidir en el aumento del número de nuevos turistas, la existencia de la AVF en Tarragona está generando un significativo cambio en el modo de transporte para acceder al destino turístico entre aquellos turistas que no era la primera vez que visitaban la Costa Dorada.

Tal como sea, la existencia de una infraestructura de transporte en el entorno de un destino turístico es una de las múltiples causas que pueden influir en la toma de decisión de los turistas de visitarlo. En nuestro caso, podemos afirmar que la disponibilidad de los servicios de AVF es tenida en cuenta en esta toma de decisiones, y que la existencia de la estación de AVF Camp de Tarragona incrementa, sin ninguna duda, la probabilidad de visitar la Costa Dorada. El resultado, a diferencia de otras aproximaciones llevadas a cabo en otros estudios, se ha determinado en base a la aplicación de un método probabilístico causal en el que se cuantifica el peso que la AVF ha tenido en la toma de decisión y no en base a una respuesta cualitativa (sí/no) a la pregunta sobre la influencia que tiene este medio de transporte en la elección del destino turístico. Los resultados, al igual que se ha demostrado en relación con las características del viaje y de la estancia de los turistas, permiten confirmar que a mayor distancia entre el lugar de procedencia y la Costa Dorada, mayor es la influencia ejercida por la estación de AVF Camp de Tarragona en la elección de este destino turístico. Por el contrario, la probabilidad de haber escogido este mismo destino, independientemente de la existencia de la estación de AVF, es mayor entre aquellos turistas procedentes de regiones más cercanas 
a la Costa Dorada y que, por consiguiente, disponen de modos de transporte alternativos para realizar el trayecto y también válidos en relación con el coste y duración del viaje.

Finalmente, consideramos que los resultados son de interés, tanto para la compañía RENFE, como para los diferentes agentes del territorio vinculados al sector turístico. Un sector que debe tomar en consideración que el perfil de los turistas españoles que utilizan los servicios de la AVF en la estación de Camp de Tarragona es muy heterogéneo, especialmente en relación con variables sensibles como son la incorporación de nuevos turistas, el tamaño de los grupos, la duración de la estancia y el tipo de alojamiento.

\section{Referencias bibliográficas}

Albalate, D. y Bel, G. (2011). «Cuando la economía no importa: auge y esplendor de la alta velocidad en España». Revista de Economía Aplicada, 55, 171-190.

Albalate, D.; Bel, G. y FaGEDA, X. (2015). «Competition and cooperation between high speed rail and air transportation services in Europe». Journal of Transport Geography, 42, 166-174.

$<$ https://doi.org/10.1016/j.jtrangeo.2014.07.003>

Alegre, J. y Pou, L. L. (2006). "The length of stay in the demand for tourism». Tourism Management, 27, 1343-1355. $<$ http://dx.doi.org/10.1016/j.tourman.2005.06.012>

Alén, E.; Nicolau, J. L.; Losada, N. y Domínguez, T. (2014). «Determinants factors of senior tourists' length of stay». Annals of Tourism Research, 49, 19-32. $<$ http://dx.doi.org/10.1016/j.annals.2014.08.002>

Anton Clavé, S.; GutiérRez, A. y Saladié, O. (2015a). «High speed rail services in a consolidated Catalan Mediterranean mass coastal destination: a causal approach». En: International Conference High Speed Rail and the City: Tourism and dynamics around stations. Paris-East University, 21-23 de enero de 2015.

Anton Clavé, S.; Saladié, O.; Cortés-Jiménez, I.; Fernandez Young, A. y YounG, R. (2015b). «How different are tourists who decide to travel to a mature destination because of the existence of a low-cost carrier route?». Journal of Air Transport Management, 42, 213-218. <https://doi.org/10.1016/j.jairtraman.2014.11.001>

BAZin, S.; BeCKerich, C. y Delaplace, M. (2010). «Desserte ferroviaire à grande vitesse, activation des ressources spécifiques et développement du tourisme: le cas de l'agglomération rémoise». Belgeo, 1-2, 65-78.

- (2013). «Desserte TGV et villes petites et moyennes. Une illustration par le cas du tourisme à Arras, Auray, Charleville Mézières et Saverne». Les Cahiers Scientifiques des Transport, 63, 33-62.

Bellet, C.; Alonso, P. y GutiérRez, A. (2012). «The High Speed Rail in the Spanish cities: urban integration and strategies for socioeconomic development». En: J. M. URENÁ (coord.). Territorial implications of High Speed Rail in Spain. Aldershot: Ashgate, 163-192.

Bellet, C. y GutiérRez, A. (2011). «Ciudad y ferrocarril en la España del siglo XXI. La integración de la alta velocidad ferroviaria en el medio urbano». Boletín de la Asociación de Geógrafos Españoles, 55, 151-179.

Bull, A. (1991). The economics of travel and tourism. Melbourne: Pitman. 
Burckhart, K. Martí-Henneberg, J. y Tapiador, F. J. (2008). «Cambio de hábitos y transformaciones territoriales en los corredores de alta velocidad ferroviaria. Resultados de una encuesta de viajeros en la línea Madrid-Barcelona». Scripta Nova. Revista Electrónica de Geografía y Ciencias Sociales, 270.

Clewlow, R. R.; Sussman, J. M. y Balakrishnan, H. (2014). «The impact of highspeed rail and low-cost carriers on European air passenger traffic». Transport Policy, 33, 136-143.

<https://doi.org/10.1016/j.tranpol.2014.01.015>

Coronado, J. M.; Garmendia, M.; Moyano, A. y Ureña, J. M. (2012). «Measuring high speed rail usefulness for tourism in Spain». En: Communication au colloque de l'ASRDLF, julio de 2012. Belfort (France), 18 p.

Cracolici, M. F. y NijKamp, P. (2008). «The attractiveness and competitiveness of tourist destinations: a study of Southern Italian regions». Tourism Management, 30, 336-344. <http://dx.doi.org/10.1016/j.tourman.2008.07.006>

Crouch, G. I. y Ritchie, R. B. (2005). «Application of the analytic hierarchy process to tourism choice and decision making: a review and illustration applied to destination competitiveness». Tourism Analysis, 10, 17-25.

Decrop, A. y Snelders, D. (2004). «Planning the summer vacation. An adaptable process». Annals of Tourism Research, 31, 1008-1030. <http://dx.doi.org/10.1016/j.annals.2004.03.004>

Delaplace, M.; Pagliara, F.; Perrin, J. y Mermet, S. (2014). "Can High Speed Rail Foster the Choice of Destination for Tourism Purpose?». Procedia-Social and Behavioral Sciences, 111, 166-175. <http://dx.doi.org/10.1016/j.sbspro.2014.01.049>

Dickinson, J. E. y PEETERS, P. (2014). «Time, tourism consumption and sustainable development». International Journal of Tourism Research, 16, 11-21. <https://doi.org/10.1002/jtr.1893>

Dobruszkes, F.; Dehon, C. y Givoni, M. (2014). «Does European high-speed rail affect the current level of air services? An EU-wide analysis». Transportation Research Part A: Policy and Practice, 69, 461-475. <https://doi.org/10.1016/j.tra.2014.09.004>

Duval, D. T. (2007). Tourism and transport: modes, networks and flows. Clevedon: Channel View Publications.

ELDRIDGE, J. D. y JONES, J.P. (1991). "Warped space: a geography of distance decay». The Professional Geographer, 43, 500-511. <http://dx.doi.org/10.1111/j.0033-0124.1991.00500.x>

ENRIGHT, M. J. y NEWTON, J. (2004). «Tourism destination competitiveness: a quantitative approach». Tourism Management, 25, 777-788. <http://dx.doi.org/10.1016/j.tourman.2004.06.008>

FACCINETTI-MANNONE, V. (2005). «La nodalité des gares TGV périphériques». Les Cahiers Scientifiques du Transport, 48, 45-58.

FeliU, J. (2012). "High-Speed Rail in European Medium-Sized Cities: Stakeholders and Urban Development». Journal of Urban Planning Development, 138, 293-302. <http://dx.doi.org/10.1061/(ASCE)UP.1943-5444.0000123>

Fernandez Young, A. y Young, R. (2008). «Measuring the effect of film and television on tourism to screen locations: a theoretical and empirical perspective». Journal of Travel \& Tourism Marketing, 24, 195-212. <http://dx.doi.org/10.1080/10548400802092742> 
Ferrer-Rosell, B.; Martínez-García, E. y Coenders, G. (2014). «Package and no-frills air carriers as moderators of length of stay». Tourism Management, 42, 114-122. <http://dx.doi.org/10.1016/j.tourman.2013.11.002>

Garmendia, M.; Ribalaygua, C. y Ureña, J. M. (2012). «High speed rail: implication for cities». Cities, 29, 26-31. <http://dx.doi.org/10.1016/j.cities.2012.06.005>

Garmendia, M.; Ureña, J. M. y Coronado, J. (2011). "Cambios en la estructura territorial debidos a nuevas conexiones de alta velocidad en territorios aislados: la provincia de Ciudad Real en España». EURE, 37, 89-115. <http://dx.doi.org/10.4067/S0250-71612011000100004>

GOKOVALI, U.; BaHAR, O. y KOZAK, M. (2007). «Determinants of length of stay. A practical use of survival analysis». Tourism Management, 28, 736-746. <http://dx.doi.org/10.1016/j.tourman.2006.05.004>

Gomes de Menezes, A.; Moniz, A. y Vieira, J.C. (2008). "The determinants of length of stay in the Azores». Tourism Economics, 14, 205-222. <http://dx.doi.org/10.5367/000000008783554866>

Gossling, S.; Hall, C. M. y Weaver, D. B. (2009). Sustainable tourism futures: perspectives on systems, restructuring and innovations. Oxon: Routledge.

GUTIÉRREZ, J. (2004). «El tren de alta velocidad y sus efectos espaciales». Investigaciones Regionales, 5, 199-224.

GutiéRreZ, A. (2009). «Alta Velocidad Ferroviaria en España y estaciones periféricas. Retos y oportunidades a la luz del caso del Camp de Tarragona». En: F. PILlET et al. (eds.). Geografía, territorio y paisaje: el estado de la cuestión. Ciudad Real: Asociación de Geógrafos Españoles, Universidad de Castilla-La Mancha, 383-400.

Gutiérrez, A. y Miravet, D. (2016). "The determinants of tourist use of public transport at the destination». Sustainability, 8, 908. <https://doi.org/10.3390/su8090908>

Gutiérrez, A.; Saladié, Ò. y Anton Clavé, S. (2018). «High-speed rail and tourism destination choice: the role and significance of the Camp de Tarragona station for passengers visiting the Costa Daurada». Boletín de la Asociación de Geógrafos Españoles, 76, 479-503. <http://dx.doi.org/10.21138/bage.2531>

Hooper, J. (2015). «A destination too far? Modelling destination accessibility and distance decay in tourism». GeoJournal, 80, 33-46. <http://dx.doi.org/10.1007/s10708-014-9536-z>

Khadaroo, J. y SeETANAH, B. (2008). «The role of transport infrastructure in international tourism development: A gravity model approach». Tourism Management, 29, 831-840. <http://dx.doi.org/10.1016/j.tourman.2007.09.005>

Lee, H. A.; Guillet, B. D.; LaW, R. y Leung, R. (2012). «Robustness of distance decay for international pleasure travelers: a longitudinal approach». International Journal of Tourism Research, 12, 409-420. <http://dx.doi.org/10.1002/jtr.861>

Lumsdon, L. y PAGE, S. J. (2004). «Tourism and transport: issues and agenda for the new millennium», Advances in Tourism Research Series. Londres: Routledge.

Masson, S. y PETIOT, R. (2009). "Can the high speed rail reinforce tourism attractiveness? The case of the high speed rail between Perpignan (France) and Barcelona (Spain)». Technovation, 29, 611-617. <http://dx.doi.org/10.1016/j.technovation.2009.05.013> 
MaZANeC, J.; WÖBER, K. y Zins, A. (2007). «Tourism destination competitiveness: from definition to explanation?». Journal of Travel Research, 46, 86-95. <http://dx.doi.org/10.1177/0047287507302389>

McKercher, B. y LEW, A. A. (2003). «Distance decay and the impact of effective tourism exclusion zones on international travel flows». Journal of Travel Research, 42, 159-165. <http://dx.doi.org/10.1177/0047287503254812>

McKercher, B.; CHAn, A. y Lam, C. (2008). "The impact of distance on international tourist movements». Journal of Travel Research, 47, 208-224. <http://dx.doi.org/10.1177/0047287508321191>

Miossec, J-M. (1977). «Un modèle de l'espace touristique». Espace Géographique, 6, 41-48. <http://dx.doi.org/10.3406/spgeo.1977.1690>

OMT (2010). Recomendaciones internacionales para estadisticas de turismo 2008. Madrid/Nueva York: Departamento de Asuntos Económicos y Sociales. Organización Mundial del Turismo. Naciones Unidas.

Ortega, E.; López, E. y Monzón, A. (2012). «Territorial cohesion impacts of high-speed rail at different planning levels». Journal of Transport Geography, 24, 130-141. <http://dx.doi.org/10.1016/j.jtrangeo.2011.10.008>

Ortuño, A.; Bautista, B.; Fernández, P.; Fernández, G.; SÁnchez, J. C. (2015). «HSR passengers profile in sun and beach tourism destinations: the case of Alicante (Spain)». En: International Conference "High Speed Rail and the City: Tourism and dynamics around stations"». Paris-Est University, 21-23 enero de 2015.

Page, S. J. y Connell, J. (2009). Tourism: a modern synthesis. Hampshire: Cengage Learning EMEA.

Pagliara, F.; La Pietra, A.; Gomez, J. y Vassallo, J. M. (2015). «High Speed Rail and the tourism market: evidence from the Madrid case study». Transport Policy, 37, $187-194$. <https://doi.org/10.1016/j.tranpol.2014.10.015>

PIRIE, G. (2009). «Distance». En: R. KITCHIN, y N. THRIFT (eds.). International encyclopedia of human geography (Vol. 1). Oxford: Elsevier.

PRIDEAUX, B. (2002). "Building visitor attractions in peripheral areas - Can uniqueness overcome isolation to produce viability?». International Journal of Tourism Research, 4, 379-389. <http://dx.doi.org/10.1002/jtr.387>

Saladié, O.; Anton Clavé, S.; Cortés-Jiménez, I.; Fernandez Young, A. y YounG, R. (2014). "La influencia de las rutas de vuelos de bajo coste en la elección del destino turístico». Cuadernos de Turismo, 34, 287-312.

SAlADIÉ, O. y Pla, E. (2015). «La mejora de la recogida selectiva de los residuos municipales atribuible a una campaña de sensibilización: un estudio de caso». Cuadernos Geográficos, 54, 64-86.

Saladié, O. y SANTOS-LaCUEVA, R. (2016). "The role of awareness campaigns in the improvement of separate collection rates of municipal Waste among university students: a causal chain approach». Waste Management, 48, 48-55. <http://dx.doi.org/10.1016/j.wasman.2015.11.037>

Saladié, O.; Anton Clavé, S. y Gutiérrez, A. (2016). «Measuring the influence of the Camp de Tarragona high-speed rail station on first-time and repeat tourists visiting a coastal destination». Belgeo, 2/2016. <https://doi.org/10.4000/belgeo.17889> 
Salmasi, L.; Celidoni, M. y Procidano, I. (2012). «Length of stay: Price and income semi-elasticities at different destinations in Italy». International Journal of Tourism Research, 14, 515-530. <https://doi.org/10.1002/jtr.1867>

Santos, J. M. Aguilera, J.; Borderías, M. P. y GonzÁlez, M.P. (2007). «La movilidad interurbana en la línea de Alta Velocidad Madrid-Sevilla: rasgos definitorios a los 10 años de su implantación». Anales de Geografía, 26, 147-165.

Thrane, C. (2012). «Tourists' length of stay: the case of international summer visitors to Norway». Tourism Economics, 18, 1069-1082. <http://dx.doi.org/10.5367/te.2012.0158>

UM, S. y CROMPTON, J. L. (1991). "Attitude determinants in tourism destination choice». Annals of Tourism Research, 17, 432-448.

Ureña, J. M.; Menerault, P. y Garmendia, M. (2009). «The high-speed rail challenge for big intermediate cities: a national, regional and local perspective». Cities, 26, 266-279.

Wang, E.; Little, B. B. y Del Homme-LitTle, B. (2012). «Factors contributing to tourists' length of stay in Dalian northeastern China - A survival model analysis». Tourism Management Perspectives, 4, 67-72. <https://doi.org/10.1016/j.tmp.2012.03.005>

WU, C. L. y CARSON, D. (2008). "Spatial and temporal tourist dispersal analysis in multiple destination travel». Journal of Travel Research, 46, 311-317. <http://dx.doi.org/10.1177/0047287506304046>

Young, R.; Fernandez Young, A. y Wu, M. (2005). «Causing tourism». En: T. Sinclair (ed.). Proceedings of the 4 th DeHaan Tourism Conference. Nottingham: Nottingham University, 32-41.

Young, R.; Fernandez Young, A.; Parkin, J. y Diamond, A. (2010). «Assessing the economic impact of culture in English market towns: a causal chain approach». Tourism Economics, 16, 925-951. <http://dx.doi.org/10.5367/te.2010.0016> 